\title{
Comparación de tres técnicas de irrigación en la remoción de hidróxido de calcio
}

\author{
Comparison of three irrigation techniques in the removal of calcium hydroxide
}

\begin{abstract}
Ruth Viviana Intriago Morales,a,b, Elida Magali Ortiz Garay ${ }^{2, a}$, Deborah Estefanía Narváez Miranda ${ }^{3, a}$, Andrés Gustavo Vega Yépez ${ }^{1, a, c, d}$, Ebingen Villavicencio Caparóó ${ }^{1,4, d, e, f}$.
\end{abstract}

\section{RESUMEN}

Objetivos: Comparar la eficacia de tres de protocolos de irrigación en la remoción de hidróxido de calcio $\left[\mathrm{Ca}(\mathrm{OH})_{2}\right]$. Material y Métodos: Mediante un diseño de estudio comparativo in vitro, se instrumentaron 106 conductos radiculares de incisivos bovinos hasta una lima de diámetro 60, la raíces se fraccionaron en dos mitades siguiendo el eje mayor del diente y se creó un surco estandarizado a $2 \mathrm{~mm}$ del agujero apical que fue rellenado con una pasta de hidróxido de calcio, luego se reensamblaron las mitades, se incubaron por 7 días y se realizaron los protocolos de irrigación: ultrasónica pasiva (PUI), sónica con Endo Activator (EA) y activación dinámica manual (MDA), donde se usó como irrigante hipoclorito de sodio $(\mathrm{NaOCl})$ al $5 \%$. Se utilizó un control negativo y positivo. Con un estereomicroscópio se observó la cantidad de residuo, las imágenes se examinaron y se les asignó una puntación de acuerdo a la escala de van der Sluis, finalmente los datos se analizaron con la prueba estadística del Chi Cuadrado. Resultados: Los porcentajes de eficacia para los protocolos de irrigación PUI, EA y MDA fueron del 87,5\%; 46,9\% y 28,1\% respectivamente, la técnica PUI fue superior a EA y MDA y obtuvo diferencias estadísticamente significativas $(\mathrm{p}<0,001)$. Conclusiones: PUI fue el método más efectivo en la remoción de $\mathrm{Ca}(\mathrm{OH})_{2}$ de los surcos simulados en los conductos radiculares.

PALABRAS CLAVE: Activación dinámica manual, Endo Activator, irrigación sónica, irrigación ultrasónica, remoción de hidróxido de calcio.

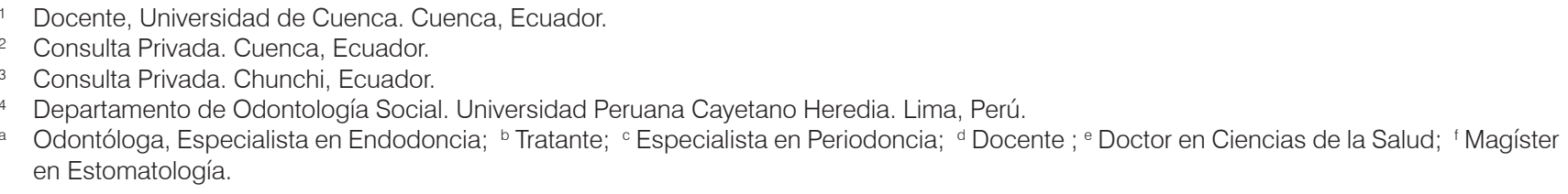




\section{SUMMARY}

Objectives: To compare efficacy of three irrigation protocols in the removal of calcium hydroxide $\left[\mathrm{Ca}(\mathrm{OH})_{2}\right]$. Materials and Methods: Through an in vitro comparative study design, 106 root canals of bovine incisors were instrumented up to a file of diameter 60 , the roots were fractioned into two halves following the major axis of the tooth and a standardized groove was created $2 \mathrm{~mm}$ from the apical foramen that was filled with a paste of calcium hydroxide, then the halves were reassembled, incubated for 7 days and the irrigation protocols were carried out: passive ultrasonic (PUI), sonic with Endo Activator (EA) and manual dynamic activation (MDA), where 5\% sodium hypochlorite $(\mathrm{NaOCl})$ was used as irrigant. A negative and positive control was used. With a stereomicroscope the amount of residue was observed, the images were examined and assigned a score according to the van der Sluis scale, finally the data were analyzed with the statistical test of Chi-square. Results: The percentages of efficacy for PUI, EA and MDA irrigation protocols were $87.5 \% ; 46.9 \%$ and $28.1 \%$ respectively, the PUI technique was superior to EA and MDA and obtained statistically significant differences $(\mathrm{p}<0.001)$. Conclusions: PUI was the most effective method in the removal of $\mathrm{Ca}(\mathrm{OH})_{2}$ from the simulated grooves in the root canals.

KEYWORDS: Manual dynamic activation, Endo Activator, sonic irrigation, ultrasonic irrigation, calcium hydroxide removal.

\section{INTRODUCCIÓN}

El hidróxido de Calcio se utiliza como una medicación intraconducto muy popular en Endodoncia, ya que ostenta una gran cantidad de beneficios y propicia respuestas biológicas favorables como: la estimulación de la formación de tejidos mineralizados, el efecto antimicrobiano, la inhibición de lipopolisacáridos bacterianos, entre otras $(1,2)$. Pese a estas ventajas su remoción del interior del conducto radicular es muy complicada (2). La persistencia de este material compromete la integridad de la obturación del conducto, provocando un sellado deficiente entre las paredes de dentina y los materiales de obturación, propiciando así la fuga apical $(3,4)$. Por lo que se han planteado varios métodos de irrigación que optimicen su eliminación durante la terapéutica endodóntica $(2,3,5)$. Existe una controversia sobre la eficacia de los métodos de irrigación en la eliminación del $\mathrm{Ca}(\mathrm{OH})_{2}$, por lo que mediante una revisión sistemática se comparó a la irrigación ultrasónica versus otros métodos de activación, pero no obtuvieron resultados concluyentes que demuestren que es superior, además se notó que las investigaciones se realizaron con pequeños tamaños muestrales (6).

El objetivo de este estudio fue hacer una comparación de la eficacia de los protocolos de irrigación: ultrasónica pasiva, sónica y agitación dinámica manual para eliminar el hidróxido de calcio de los surcos creados en el interior de los conductos radiculares de incisivos bovinos.

\section{MATERIAL Y MÉTODOS}

El diseño de estudio fue comparativo in vitro y transversal. Se usaron incisivos de bovino por su parecido histológico y morfológico a los incisivos humanos, los mismos que se obtuvieron en el Camal Municipal de la ciudad de Cuenca de animales destinados al faenamiento para el consumo humano, cumpliendo así los parámetros bioéticos. Se realizó el cálculo del tamaño muestral utilizando la fórmula de muestreo para comparar dos proporciones, con un nivel de confianza del $95 \%$ y se obtuvo 32 dientes por grupo de estudio $(3,7)$.

\section{Procedimiento y técnicas}

Se seleccionaron 106 incisivos bovinos, tras su extracción se sumergieron por 24 horas en una solución de $\mathrm{NaOCl}$ al $5 \%$, lo que disolvió los residuos de tejido blando que estaban adheridos a las raíces, en seguida se rasparon manualmente con una cureta (8) y finalmente se almacenaron en agua destilada (3).

Mediante un disco de diamante se decoronaron los incisivos, con lo que se estandarizó su tamaño a $16 \mathrm{~mm}$ (9). Se comprobó su permeabilidad con una lima K \# 10 y se determinó su longitud de trabajo (LT) insertando una lima $\mathrm{K} \# 15$ hasta que se observe a través del agujero apical y restando $1 \mathrm{~mm}$ a esta medición. Con la técnica de fuerzas balanceadas y a la LT se instrumentaron las raíces utilizando limas Flexofiles (Dentsply Maillefer) hasta un diámetro 60, en el caso de que los dientes superaran este tamaño se desecha- 
ban de la investigación. La irrigación se realizó entre lima y lima con una jeringa de $3 \mathrm{~mm}$ y una aguja de $30 \mathrm{G}$ (Navitip, Ultradent) que se introdujo a $2 \mathrm{~mm}$ de la LT y con $2 \mathrm{ml} \mathrm{NaOCl}$ al 5\% como solución irrigadora (10).

Se prepararon dos surcos siguiendo el eje longitudinal de la raíz a cada lado de la misma empleando un disco de diamante sin llegar a tocar la luz del conducto y luego con un cincel se la dividió en dos mitades (10). Si la raíz tenía agujeros visibles al momento del reensamblaje se desechaba.

Para simular una irregularidad en el interior del conducto se creó en una de las mitades un surco estandarizado de 0,2 $\mathrm{mm}$ de ancho, $4 \mathrm{~mm}$ de longitud y $0,5 \mathrm{~mm}$ de profundidad a $2 \mathrm{~mm}$ del agujero apical, mediante un disco de diamante de $0,2 \mathrm{~mm}$ de grosor, para esto y con apoyo de un calibrador manual se trazó con lápiz una marca donde iba el surco, luego se utilizó el disco que fue accionado por una pieza de mano (NSK) de baja velocidad hasta que la marca desaparezca y finalmente se removió el debris del surco (9).

Se procedió al llenado de los surcos con una pasta de $\mathrm{Ca}(\mathrm{OH})_{2}$ (CALCIFAR-P), se compactó el material accionando una punta ultrasónica por 10 segundos (11) y se retiraron los excesos con un microbrush; en el grupo control negativo (5 muestras) no se realizó este procedimiento. A continuación se reensamblaron las raíces con cera pegajosa y alambre (12), se sellaron con algodón y cavit (ESPE) para finalmente incubarlas a $37^{\circ} \mathrm{C}$ al $100 \%$ de humedad por 7 días (3).

En el grupo control positivo (5 muestras) no se realizó ningún protocolo de irrigación (3). Los 96 especímenes restantes se numeraron, se etiquetaron y se los asignó aleatoriamente a los 3 grupos experimentales (32 muestras c/u). Una vez que se retiró el algodón y el cavit, se realizaron los protocolos de irrigación como se detalla a continuación:

Con una jeringa de $3 \mathrm{~mm}$ y una aguja de $30 \mathrm{G}$ (Navitip; Ultra-Dent) insertada a $2 \mathrm{~mm}$ de la LT se suministró a los conductos $8 \mathrm{ml}$ de $\mathrm{NaOCl}$ al $5 \%$ a una velocidad de flujo de $2 \mathrm{ml} / 20 \mathrm{seg}$. La activación del irrigante se hizo por 1 minuto y cada 20 segundos se realizó una pausa para el recambio $2 \mathrm{ml}$ de $\mathrm{NaOCl}(9)$.

Grupo 1: Irrigación Ultrasónica Pasiva (PUI): Se realizó ligeros movimientos de entrada y salida en el conducto a $1 \mathrm{~mm}$ de la LT con una lima ultrasónica IRRI K tamaño 15; 0,02 (VDW) accionada por un equipo ultrasónico (ACTEON SATELEC) a un nivel de potencia 4 , lo que permitió la activación pasiva del irrigante, sin tocar las paredes del conducto, es decir vibrando libremente (4).

Grupo 2: Irrigación Sónica, Endo Activator (EA): Se activó el irrigante introduciendo en el conducto a 2 mm de la LT una punta tamaño 25; 0,04 accionada por un dispositivo sónico Endo Activator y se realizó ligeros movimientos de entrada y salida (3).

Grupo 3: Activación Dinámica Manual (MDA): Se ajustó un cono de gutapercha a la LT y luego se agitó el $\mathrm{NaOCl}$ insertándolo y realizando movimientos verticales ascendentes y descententes (2 a $3 \mathrm{~mm})(13,14)$ a una velocidad de 3 golpes por segundo (13). Con el fin de modular la frecuencia de golpes se hizo una calibración intrasujeto acorde al índice Kappa previo a este procedimiento, se consiguió un valor kappa $>0.8$, es decir un nivel de concordancia muy buena.

Luego de realizados los protocolos de irrigación los conductos se secaron con puntas de papel \# 60 (15) y se separaron las mitades para observar el residuo de $\mathrm{Ca}(\mathrm{OH})_{2}$ mediante un estereomicroscopio (Boeco Germany) a una magnificación de 40X, colocando los especímenes en un mismo sitio. Se consiguieron imágenes digitales para analizar la remoción del material de los surcos de acuerdo a las puntuaciones de la escala van der Sluis (figura 1) en donde 0 significa surco vacío, 1 menos de la mitad del surco con $\mathrm{Ca}(\mathrm{OH})_{2}$, 2 más de la mitad del surco con $\mathrm{Ca}(\mathrm{OH})_{2}$ y 3 surco totalmente lleno con $\mathrm{Ca}(\mathrm{OH})_{2}(5)$.

Se utilizaron 30 imágenes al azar para calibrar según el índice Kappa de Cohen a 2 observadores y de esta manera puedan identificar cuando se trata de la puntuación $0,1,2$ o 3 . Tras 24 horas nuevamente recibieron 48 imágenes al azar y se registraron los datos. Se generó una base de datos y se procedió al análisis de frecuencias y porcentajes tanto de la variable remoción como eficacia, luego se realizó la prueba estadística del Chi Cuadrado para comparar porcentajes de los niveles de remoción y porcentajes de eficacia y no eficacia entre los grupos de estudio. Se empleó un nivel de significancia del $95 \%$. 


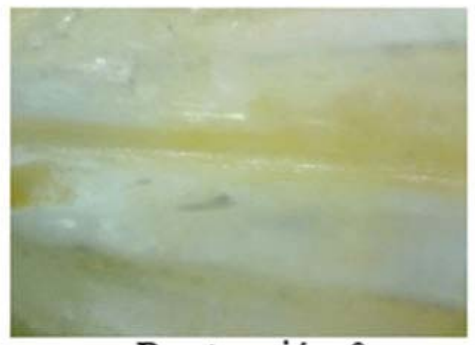

Puntuación 0

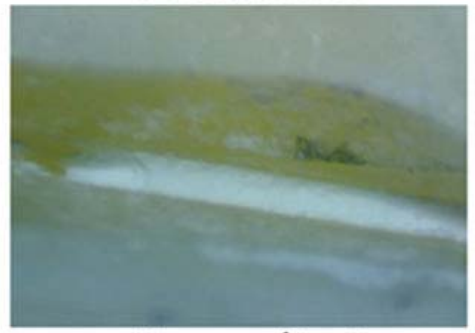

Puntuación 2

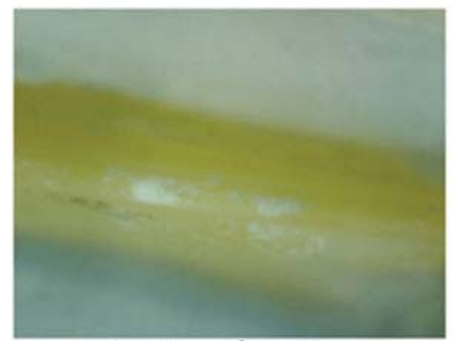

Puntuación 1

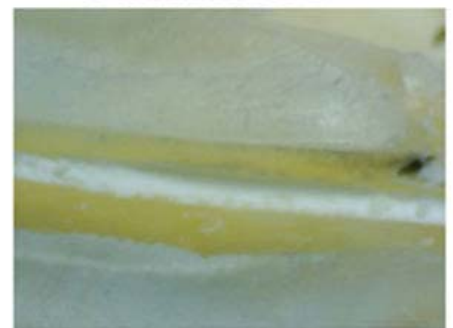

Puntuación 3

Figura 1. Niveles de Remoción de $\mathrm{Ca}(\mathrm{OH})_{2}$, magnificación 40X.

\section{RESULTADOS}

El nivel de concordancia interobservador kappa para la observación de la remoción de hidróxido de calcio fue de 0,85 (muy bueno).

Se observó diferencias estadísticamente significativas entre todos los grupos experimentales y con los grupos control negativo y positivo $(\mathrm{p}<0,01)$ en cuanto a los niveles de remoción del $\mathrm{Ca}(\mathrm{OH})_{2}$.

Se determinó que en un $43.8 \%$ la técnica PUI alcanzó la remoción total de $\mathrm{Ca}(\mathrm{OH}) 2$, en comparación al grupo EA y MDA con un porcentaje de 15,6\% y 3,1\% respectivamente, lo que indica que entre los protocolos evaluados en esta investigación PUI logró remo- ver significativamente más hidróxido de calcio de los $\operatorname{surcos}(\mathrm{p}<0,01)$ (Tabla 1$)$.

Las técnicas PUI, EA y MDA obtuvieron porcentajes de eficacia del $87,5 \% ; 46,9 \%$ y $28,1 \%$ respectivamente como se aprecia en el gráfico 1 , por lo que se establece que el método de irrigación más eficaz en la eliminación del $\mathrm{Ca}(\mathrm{OH})_{2}$ fue el protocolo PUI, siendo estadísticamente diferente a las otras técnicas probadas.

Al hacer la comparación entre grupos sobre la remoción y eficacia de eliminación del hidróxido de calcio se observa que PUI es mejor y estadísticamente diferente al protocolo EA (Chi Cuadrado $\mathrm{p}<0,01)$.

Tabla 1. Distribución de los especímenes en los niveles de remoción de $\mathrm{Ca}(\mathrm{OH})_{2}$ luego de realizados los protocolos de irrigación.

\begin{tabular}{|c|c|c|c|c|c|c|c|c|c|c|}
\hline \multirow[b]{3}{*}{ GRUPO } & \multicolumn{9}{|c|}{ NIVELES DE REMOCIÓN } & \\
\hline & \multicolumn{2}{|c|}{$\mathbf{0}$} & \multicolumn{2}{|c|}{1} & \multicolumn{2}{|c|}{2} & \multicolumn{2}{|c|}{3} & & \\
\hline & $\mathrm{n}$ & $\%$ & $\mathrm{n}$ & $\%$ & $\mathrm{n}$ & $\%$ & $\mathrm{n}$ & $\%$ & $\mathrm{n}$ & $\%$ \\
\hline PUI & 14 & 44 & 14 & 43.8 & 4 & 12.5 & 0 & 0 & 32 & 100 \\
\hline EA & 5 & 16 & 10 & 31.3 & 16 & 50 & 1 & 3.1 & 32 & 100 \\
\hline MDA & 1 & 3.1 & 8 & 25 & 14 & 43.8 & 9 & 28.1 & 32 & 100 \\
\hline Total & 20 & 21 & 32 & 33.3 & 34 & 35.4 & 10 & 10.4 & 96 & 100 \\
\hline
\end{tabular}

Prueba Chi cuadrado $\mathrm{p}<0,01$ 


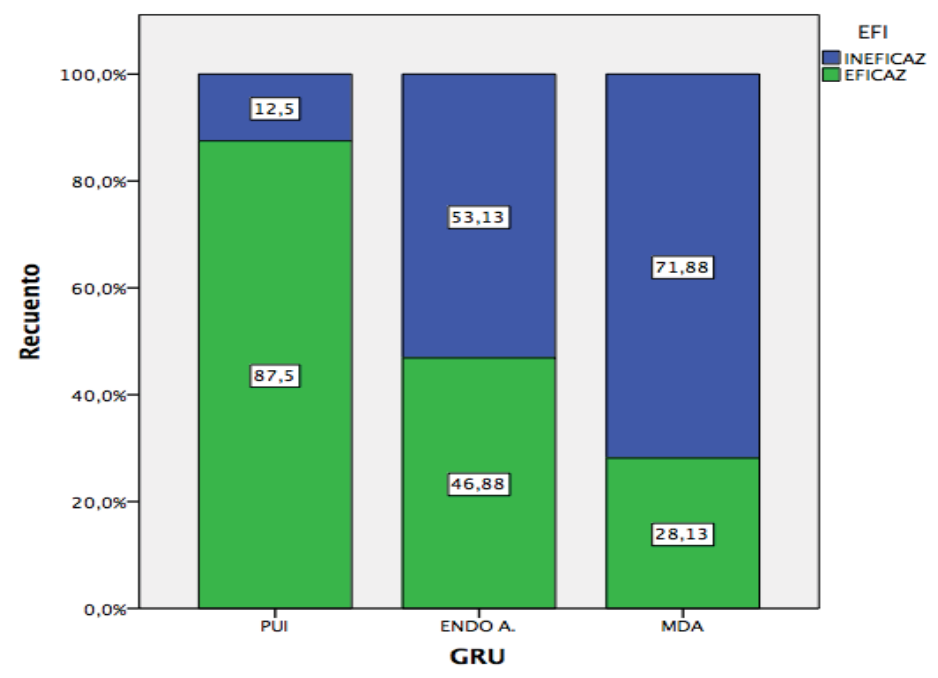

Gráfico 1. Eficacia de los grupos experimentales en la remoción de remoción de $\mathrm{Ca}(\mathrm{OH})_{2}$

Al contrastar los protocolos PUI y MDA en relación a los niveles de remoción y eficacia, se establece que son estadísticamente diferentes (Chi Cuadrado $\mathrm{p}<0,01$ )

Cuando se comparó la remoción de $\mathrm{Ca}(\mathrm{OH})_{2}$ entre los grupos EA y MDA se determinó que poseen una diferencia estadísticamente significativa (Chi Cuadrado $p=0,024)$, sin embargo al comparar su eficacia se observa que no son estadísticamente diferentes (Chi Cuadrado $\mathrm{p}=0,098$ ).

\section{DISCUSIÓN}

La técnica PUI facilita una óptima limpieza y desinfección de los conductos, debido a la cavitación hidrodinámica y transmisión acústica que permite la formación e implosión de burbujas (16), este efecto se evidencia en este estudio donde la eficacia de PUI fue del $87,5 \%$, muy superior a la técnica EA con un $46,9 \%$ y a MDA con un $28,1 \%$.

El presente es un estudio in vitro lo que se considera una limitación, sin embargo hay que mencionar que este tipo investigaciones son la base para el entendimiento de muchos procesos, nos permiten conocer las propiedades físicas, mecánicas y biológicas de materiales dentales y de los tejidos duros y blandos, por lo que los estudios in vitro son parte integral de la toma de decisiones clínicas.

Capar et al., tuvieron resultados parecidos a los de este estudio, observaron que el 100\% de las muestras estuvieron en el nivel de remoción 0 y 1 y en este estudio lo hicieron el $87,5 \%$ (4).

En otra investigación PUI fue el protocolo con el menor número de muestras en el nivel 3 (15\%), cuando se lo comparó con la agitación con jeringa y la lima XP-endo (17). En el presente estudio la técnica PUI no tuvo muestras en el nivel 3, es decir que no dejó ningún surco lleno con hidróxido de calcio.

En dos investigaciones $(18,19)$ evaluaron varias técnicas entre ellas la ultrasónica en cuanto a la eliminación de hidróxido de calcio de conductos radiculares; pese a las diferencias metodológicas con este estudio observaron que PUI deja menos remanente del material, al igual que en la presente investigación $(18,19)$.

Topçuoğlu et al. reportaron datos similares a los de este estudio, con un porcentaje de muestras para el grupo PUI del $50 \%$ en el nivel 0 y del $40 \%$ en el 1 y para el grupo EA un 20\% tanto en el nivel 0 como en el 1 (3); en esta investigación se ubicaron en el grupo PUI un $48,3 \%$ de muestras tanto en el nivel 0 como en el 1 , mientras que en el grupo EA un 15,6\% de las muestras estuvieron en el nivel 0 y un $31,3 \%$ en el nivel 1 .

Otro estudio evaluó la cantidad de residuo de $\mathrm{Ca}(\mathrm{OH})_{2}$ con microtomografía computarizada en raíces mesiales de molares inferiores y obtuvieron valores de $85,7 \%$ para PUI y de $71,5 \%$ para el EA, equiparándose nuevamente los resultados con los de esta investigación, e indicando la supremacía de PUI en relación a EA (20). 
También se encuentra coincidencia con otros estudios $(21,22)$. En uno de ellos (21) se realiza la visualización con un microscopio óptico y la cuantificación del residuo con el software Micrometrics, se reportaron porcentajes de $28,3 \%$ para PUI y $44,5 \%$ para EA (21). En la otra investigación (22) donde utilizaron un estereomicroscopio y examinaron el remanente por conteo de pixeles en un ordenador, informaron valores similares del $24 \%$ para PUI y del $54 \%$ para EA. Se puede apreciar las diferencias metodológicas con este estudio pero existe un parecido en los resultados que ratifica que la técnica ultrasónica es más efectiva que la sónica.

La superioridad de la técnica PUI en relación al protocolo EA se ha demostrado en varias investigaciones como en la presente, sin embargo hay otros estudios $(2,8,23,24)$ que no encuentran diferencias estadísticamente significativas entre estos métodos e incluso en dos de ellos $(8,24)$ la irrigación sónica tuvo ligera ventaja en relación a la ultrasónica, sin embargo su metodología fue diferente ya que usaron segundos molares inferiores con conductos en C en la una (24) y en la otra raíces mesiales curvas de molares (8), la razón de estos resultados fue atribuida a la flexibilidad de las puntas del EA ideales en conductos curvos (8).

Por otra parte evaluaron la eficacia de diferentes técnicas como MDA en la remoción de hidróxido de calcio utilizando bloques de titanio con surcos simulados (25), se observó que este método no fue el idóneo para el debridamiento del material, lo que coincide con esta investigación ya que tan solo 1 espécimen $(3,1 \%)$ estuvo libre de residuos (nivel 0$)$.

En conclusión, ningún método de irrigación de los estudiados logró la remoción absoluta del $\mathrm{Ca}(\mathrm{OH})_{2}$. Todos los grupos de este estudio fueron estadísticamente diferentes. El protocolo PUI demostró ser la técnica más eficaz. La activación dinámica manual no consiguió una remoción ideal del material y fue el método menos efectivo de los evaluados. La utilización del hidróxido de calcio como medicación intraconducto debe complementarse con un excelente protocolo de irrigación que permita removerlo lo más eficazmente posible.

\section{Correspondencia:}

Viviana Intriago

Correo electrónico: vivianaintriago_83@hotmail.com

\section{REFERENCIAS BIBLIOGRÁFICAS}

1. Mohammadi Z, Dummer PMH. Properties and applications of calcium hydroxide in endodontics and dental traumatology. Int Endod J. 2011;44(8):697-730.

2. Böttcher DE, Rahde N de M, Grecca FS. Calcium hydroxide removal: effectiveness of ultrasonic and manual techniques. Rev Odonto Ciênc. 2012;27(2):152-5.

3. Topçuoğlu HS, Düzgün S, Ceyhanlı KT, Aktı A, Pala $\mathrm{K}$, Kesim B. Efficacy of different irrigation techniques in the removal of calcium hydroxide from a simulated internal root resorption cavity. Int Endod J. 2015;48(4):309-16.

4. Capar ID, Ozcan E, Arslan H, Ertas H, Aydinbelge HA. Effect of different final irrigation methods on the removal of calcium hydroxide from an artificial standardized groove in the apical third of root canals. J Endod. 2014;40(3):451-4.

5. van der Sluis LWM, Wu MK, Wesselink PR. The evaluation of removal of calcium hydroxide paste from an artificial standardized groove in the apical root canal using different irrigation methodologies. Int Endod J. 2007;40(1):52-7.

6. Ethem-Yaylali I, Kececi AD, Ureyen-Kaya B. Ultrasonically activated irrigation to remove calcium hydroxide from apical third of human root canal system: A systematic review of in vitro studies. J Endod. 2015;41(10):1589-99.

7. Villavicencio E. El tamaño muestral para la tesis. ¿Cuántas personas debo encuestar? Odontol Act Rev Científica. 7 2018;1(3):71-6.

8. Cid T, Castillo JM, Echeverri D. Sonic versus ultrasonic activation in calcium hydroxide removal in curved canals: an in-vitro study. J Oral Res.2014;3(4):237-43.

9. Martins A, Abreu R, Santini MF, et al. Effectiveness of final irrigant protocols for debris removal from simulated canal irregularities. J Endod. 2014;40(12):2009-14.

10. Lee S-J, Wu M-K, Wesselink PR. The effectiveness of syringe irrigation and ultrasonics to remove debris from simulated irregularities within prepared root canal walls. Int Endod J. 2004;37(10):672-8.

11. Sisli SN, Ozbas H. Comparative micro-computed tomographic evaluation of the sealing quality of ProRoot MTA and MTA Angelus apical plugs placed with various techniques. J Endod. 2017;43(1):147-51.

12. van der Sluis LWM, Gambarini G, Wu MK, Wesselink PR. The influence of volume, type of irrigant and flushing method on removing artificially placed dentine debris from the apical root canal during passive ultrasonic irrigation. Int Endod J. 2006;39(6):472-6.

13. Basrani B. Endodontic Irrigation-Chemical disinfection of the root. Switzerland: Springer International Publishing; 2015.

14. Jiang L-M, Lak B, Eijsvogels LM, Wesselink P, van der Sluis LWM. Comparison of the cleaning effica- 
cy of different final irrigation techniques. J Endod. 2012;38(6):838-41.

15. Rödig T, Vogel S, Zapf A, Hülsmann M. Efficacy of different irrigants in the removal of calcium hydroxide from root canals. Int Endod J. 2010;43(6):519-27.

16. Gu L, Kim JR, Ling J, Choi KK, Pashley DH, Tay FR. Review of contemporary irrigant agitation techniques and devices. J Endod. 2009;35(6):791-804.

17. Wigler R, Dvir R, Weisman A, Matalon S, Kfir A. Efficacy of XP-endo finisher files in the removal of calcium hydroxide paste from artificial standardized grooves in the apical third of oval root canals. Int Endod J. 2017;50(7):700-5.

18. Kenee DM, Allemang JD, Johnson JD, Hellstein J, Nichol BK. A quantitative assessment of efficacy of various calcium hydroxide removal techniques. J Endod. 2006;32(6):563-5.

19. Taşdemir T, Celik D, Er K, Yildirim T, Ceyhanli KT, Yeşilyurt C. Efficacy of several techniques for the removal of calcium hydroxide medicament from root canals. Int Endod J. 2011;44(6):505-9.

20. Wiseman A, Cox TC, Paranjpe A, Flake NM, Cohenca N, Johnson JD. Efficacy of sonic and ultrasonic activation for removal of calcium hydroxide from mesial canals of mandibular molars: a microtomographic study. J Endod. 2011;37(2):235-8.
21. Gallardo L, Javier F, López S, Teresa AE. Comparación de la remoción de hidróxido de calcio con irrigación sónica versus ultrasónica: evaluación microscópica. Tesis de cirujano dentista. Santiago, Chile: Universidad Andrés Bello; 2015 (Fecha de acceso 6 de diciembre del 2016) Disponible en: http://repositorio.unab.cl/xmlui/handle/ria/2700

22. Arslan H, Akcay M, Capar ID, Saygili G, Gok T, Ertas H. An in vitro comparison of irrigation using photon-initiated photoacoustic streaming, ultrasonic, sonic and needle techniques in removing calcium hydroxide. Int Endod J. 2015;48(3):246-51.

23. Khaleel H, Al-Ashaw A, Yang Y, Pang A, Ma J. Quantitative comparison of calcium hydroxide removal by endoactivator, ultrasonic and protaper file agitation techniques: An in vitro study. J Huazhong Univ Sci Technolog Med Sci. 2013;33(1):142-5.

24. Ma JZ, Shen Y, Al-Ashaw AJ, et al. Micro-computed tomography evaluation of the removal of calcium hydroxide medicament from $\mathrm{C}$-shaped root canals of mandibular second molars. Int Endod J. 2015;48(4):333-41.

25. Goode N, Khan S, Eid AA, Niu L, Gosier J, Susin LF, et al. Wall shear stress effects of different endodontic irrigation techniques and systems. J Dent. 2013;41(7):636-41.

Recibido: 12-05-2018

Aceptado: 16-08-2018 